\title{
Elastic and Conductive Properties of Plasma-Sprayed Ceramic Coatings in Relation to Their Microstructure: An Overview
}

\author{
Igor Sevostianov and Mark Kachanov
}

(Submitted February 2, 2009; in revised form April 17, 2009)

\begin{abstract}
This article focuses on micromechanics-based models that explicitly express the elastic and conductive properties of plasma-sprayed ceramic coatings in terms of relevant microstructural parameters. These parameters reflect, in an integral way, the density and the orientation distribution of microcracks; they apply to strongly oblate pores as well. On the other hand, the porosity parameter usually plays a secondary role. Partial contacts between crack faces-a factor of major importance-are reflected via appropriate reduction of crack densities. The effect of various "irregularities" of crack shapes is discussed. Case studies of YSZ coatings demonstrate how the micromechanics-based modeling can be used and directly interfaced with $2-D$ image data.
\end{abstract}

Keywords coating, conductivity, cross-property, elasticity, plasma sprayed

\section{Introduction}

The present review discusses the elastic stiffness of plasma-sprayed ceramic coatings and their thermal conductivity in relation to their microstructure. We focus on explicit relations between the said properties and relevant microstructural features.

Plasma-sprayed ceramic coatings have a lamellar microstructure consisting of elongated, flat-like splats of diameters between 100 and $200 \mu \mathrm{m}$ and thicknesses between 2 and $10 \mu \mathrm{m}$, formed by a rapid solidification (Fig. 1a). The porous space comprises "irregular" mixture of cracks and pores of diverse shapes (Fig. 1b). Their orientation distribution is usually nonrandom (with tendency to be either parallel or normal to the substrate) resulting in noticeable anisotropy. The problem arises of their proper quantitative characterization, i.e., of identification of the argument of the function

effective property $=f(\underbrace{\text { microstructural parameter }}_{?})$

(Eq 1.1)

prior to discussing possible forms of this function.

Igor Sevostianov, Department of Mechanical Engineering, New Mexico State University, PO Box 30001, Las Cruces, NM 88003; and Mark Kachanov, Department of Mechanical Engineering, Tufts University, Medford, MA 02155. Contact e-mails: igor@ nmsu.edu and mark.kachanov@tufts.edu.
The basic requirement to the proper parameter is that it must represent individual defects according to their actual contributions to the considered property. For example, crack density parameter takes the individual crack contributions proportionally to crack sizes cubed, since this corresponds to their contributions to the effective compliance and to the conductivity.

Violation of this basic requirement may lead to inconsistencies. For illustration, we consider a family of strongly oblate pores. Their contributions to the effective compliance are almost independent of their aspect ratios, as long as they are below 0.1 (see the discussion in Sect 2). Let us assume that we express the effective compliance in terms of volume fraction of the pores (porosity $P$ ). Then, if the aspect ratios are changed, say, from 0.05 to 0.1 this would increase $P$ by a factor of two, but the effective compliance will remain almost unchanged. If, on the other hand, we increase the number of pores by a factor of two and reduce their aspect ratios by the same factor, $P$ will remain the same, but pore compliances will increase several times. In other words, the function (1.1) will not be single valued, if its argument is $P$. If, however, we recognize that strongly oblate pores are almost identical to cracks in their effect on the linear elastic properties, and identify crack density as the proper argument of (1.1), these difficulties are not encountered.

For complex microstructures, the identification of such parameters is a challenging problem. In the general context of materials science, this problem was reviewed in Ref 1.

Quantitative characterization of microstructures-i.e., identification of the proper microstructural parameters-depends on the physical property considered. For example, parameters that control the elastic and the conductive properties are largely similar, but, in cases of overall anisotropy, they are not identical. Further, if the conductivity of the gas filling the pores is to be taken into 

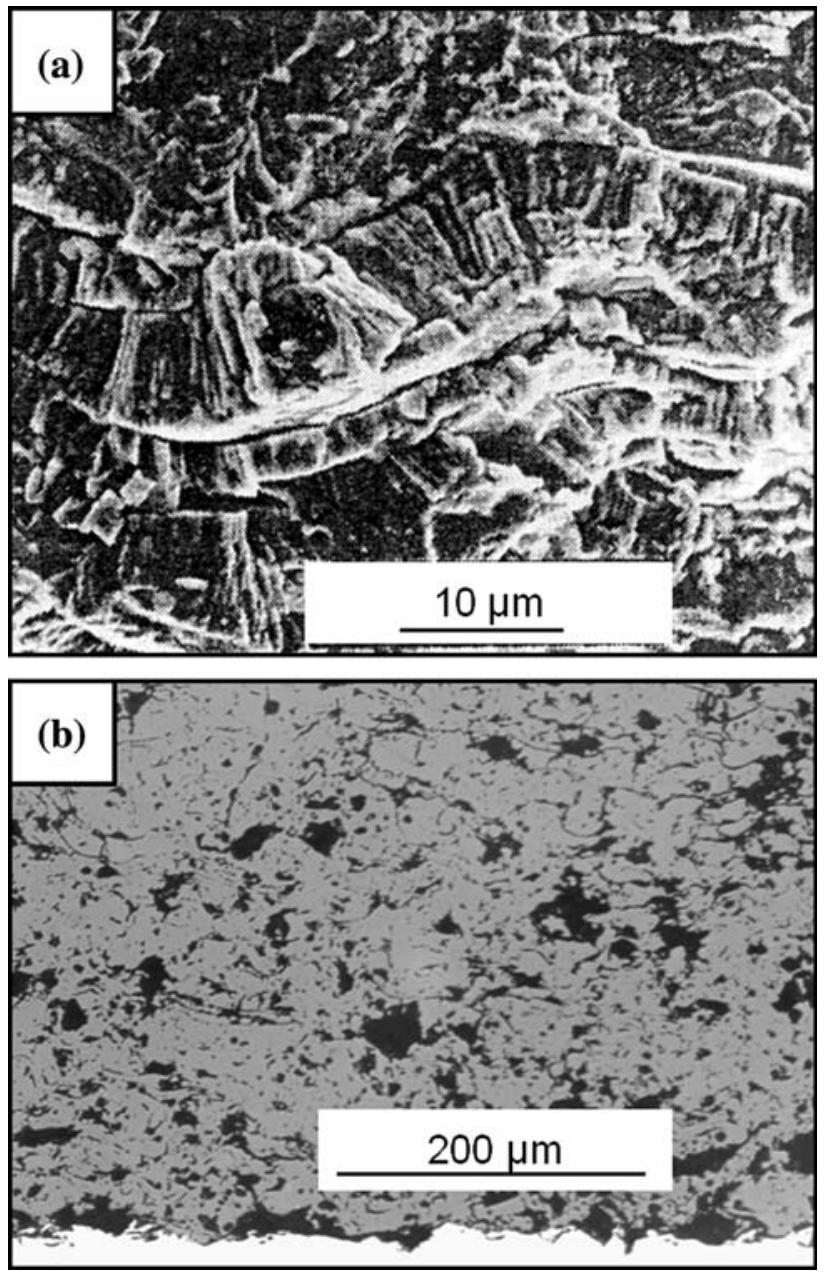

Fig. 1 Typical microstructure of plasma-sprayed ceramic coatings at different magnifications

account, the crack opening-albeit small-may become important, and this would require modification of the microstructural parameter. Importantly, the microstructural parameters that control the permeability, or fracture, may be entirely different from the ones relevant to the elastic/conductive properties.

We focus on the three-dimensional modeling. As far as 2-D models and computations are concerned, they are of qualitative and illustrative-but not quantitative-value. For example, the compliance/conductivity contributions of cracks and pores scale as their sizes cubed in 3-D, but squared in 2-D, and this difference is of major consequence for mixtures of defects of diverse sizes.

Remark We consider the linear elastic effective properties. This may present a limitation in the case of narrow, crack-like pores in the field of compressive stresses: if the latter are high enough to close some of the pores, the response is nonlinear (we refer to the review, Ref 2, for a discussion of nonlinear effects). Yet another limitation concerning conductivity is that cracks and pores are treated as ideal insulators, neglecting heat transfer across them, due to radiation or conductivity of the gas.

Three different approaches to modeling of microstructure of plasma-sprayed coatings have been suggested in literature:

A. Treating contacts between splats (that alternate with much larger no-contact zones) as dominant elements of the microstructure; the effective properties are then controlled by appropriate contact characteristics. Such modeling was developed in the context of conductivity (Ref 3); it was refined and extended to the elastic properties in a number of subsequent works; for their overview, we refer to Ref 4. One limitation of these models is that the contacts were considered as noninteracting ones, whereas interactions between contacts are generally strong and have substantial effect on the overall properties (Ref 5). We also mention that the assumption of smallness of contact areas may or may not correspond to the coating microstructure; for example, the data in Ref 6 show that contact areas may be comparable to no-contact ones. Yet another limitation concerns anisotropy: a full set of anisotropic effective constants was not given in the mentioned works. An important observation is that the crossproperty connections discussed in Sect 4 imply that models for the conductive and the elastic properties should be compatible. Such compatibility is not always clear in models of this kind (see Ref 4).

B. Treating pores and microcracks as dominant features of the microstructure; parameters of their concentration are then the controlling microstructural parameters. In Ref 7 and 8 , it is suggested to represent the porous space by two families of oblate pores, of perfectly vertical and perfectly horizontal orientations; a similar model was developed in Ref 9. One shortcoming of these models is that the effective elastic constants were expressed in terms of volume fractions of the two families (rather than crack densities). This leads to difficulties discussed above, and translates into very high sensitivity of the effective constants to the exact values of aspect ratios-a parameter that in addition to being unimportant may not be known. In addition, it is unclear how to define aspect ratios for pores of irregular shapes. In Ref 10, two families of microcracks-perfectly vertical and perfectly horizontal, plus spherical pores-were treated as the main microstructural elements, and the effective elastic constants were expressed in terms of crack density and porosity. The latter model did not consider the orientation scatter (that is, typically, substantial); this factor was accounted for in Ref 11-13.

The above-mentioned works did not take into account the presence of partial contacts between crack faces-the factor of major importance producing very strong effect on the elastic and conductive properties. This limitation 
makes it difficult to interface the mentioned works with actual image data.

In fact, approaches A and B are not mutually exclusive; on the contrary, they represent two equivalent viewpoints of the same microstructure provided approach B incorporates the effect of contacts between crack faces. Indeed, let us consider a crack with contacts. Its stiffness (the stress required to produce a given displacement of crack faces) is a sum of the stiffnesses provided by the contacts and by the crack itself; the latter stiffness is inversely proportional to the linear size of the crack (its radius, for the circular crack). As discussed in Sect 2, contacts produce very strong stiffening effect-to the extent that the stiffness of the crack itself can be neglected-except for the case when the crack is sufficiently small. Then, the crack can be viewed as an interface between two rough surfaces formed by multiple contacts, and contact-related characteristics constitute proper microstructural parameters.

This duality was discussed in the computational study (Ref 14), using the example of two periodic microstructures: one formed by isolated contacts (with interconnected no-contact zone) and another one by isolated coplanar cracks (Fig. 2). The conductances of the two arrangements were found to be relatively close if the total contact area was the same (this result applies to the stiffnesses as well, due to the cross-property connection). These computations suggest, in a qualitative manner, that the approaches $\mathrm{A}$ and $\mathrm{B}$ are equivalent, although a quantitative equivalence between the contact characteristics and the density of coplanar cracks may be difficult to establish.

C. The crack-based approach B appears to be preferable since it provides a consistent framework for describing anisotropy due to nonrandom crack orientations. Such modeling-which bridges the two approaches by reflecting the effect of contacts via appropriate reduction of the crack density-was developed in Ref 15 and 16. Their model is the focus of the present review. The discussion of the model is followed by case studies of alumina and YSZ coatings that demonstrate how the model can be used and directly interfaced with image data.

\section{Quantitative Characterization of Microstructure of the Plasma-Spray Coatings in the Context of Elastic Properties}

We view the coating as a continuous material that contains pores and cracks with contacts between their

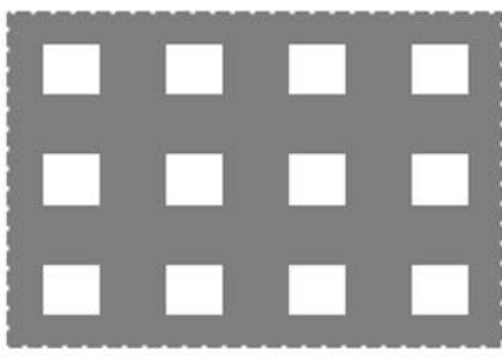

Isolated cracks

Isolated contacts

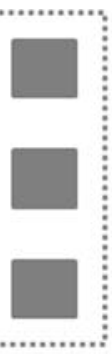

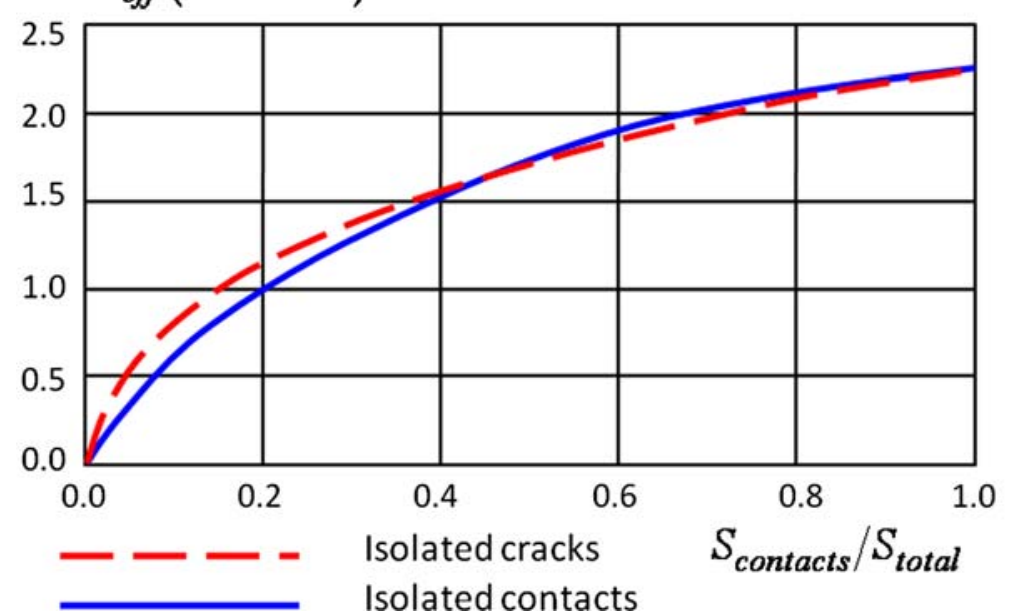

Fig. 2 Results of computational study (Ref 14) for conductivity across the interfaces with different periodic microstructures-isolated contacts and isolated cracks - as a function of relative area of contacts. Note closeness of the two curves 
faces. In this section, we discuss quantitative characterization of pores and cracks, with the account of partial contacts between crack faces and other "irregularities" of crack shapes. This means identification of the microstructural parameters that represent individual defects in accordance with their actual contributions to the elastic properties, with the account of the mentioned factors. The effective elastic properties should then be expressed in terms of such parameters, as is done in Sect 3.

The sizes of cracks and pores are very diverse: they vary from very small ones, single microns or smaller, to 10-30 $\mu \mathrm{m}$ for pores and to $100-300 \mu \mathrm{m}$ for cracks. As discussed below, only the largest defects need to be taken into account, as far as the effective elastic and conductive properties are concerned. The overall porosity depends on processing parameters and is, typically, within $10-15 \%$; it appears to be due mainly to slightly open cracks, and not pores, in view of an order-of-magnitude difference in sizes of the largest cracks and largest pores.

In the context of the effective elastic properties, the key quantity is the compliance contribution of a defect; the extra compliance $\Delta S_{i j k l}$ due to multiple defects is a sum, over a representative volume $V$, of the individual defect contributions. We start with comparing the compliance contributions of cracks and pores. We consider a circular crack of radius $a$ under uniaxial loading $\sigma_{11}$ in the direction $x_{1}$ normal to the crack. We also consider a spherical pore of radius $a$ under uniaxial loading $\sigma_{11}$. Their strain contributions $\Delta \varepsilon_{11}$ (per volume $V$ ) are as follows (Ref 17):

$$
\begin{aligned}
\Delta \varepsilon_{11}^{\text {crack }} & =\underbrace{\frac{16\left(1-v_{0}^{2}\right)}{3}}_{4.84 \text { at } v_{0}=0.3} \frac{a^{3}}{V} \frac{\sigma_{11}}{E_{0}}, \\
\Delta \varepsilon_{11}^{\text {pore }} & =\underbrace{\frac{2 \pi\left(1-v_{0}\right)\left(9+5 v_{0}\right)}{7-5 v_{0}}}_{8.40 \text { at } v_{0}=0.3} \frac{a^{3}}{V} \frac{\sigma_{11}}{E_{0}}
\end{aligned}
$$

(hereafter, $E_{0}$ and $v_{0}$ denote the Young's modulus and Poisson's ratio of the bulk material). These formulas (without the multiplier $\sigma_{11}$ ) give the contributions of the defects to the overall compliance $S_{1111}$.

Importantly, the contributions of both types of defects scale as $a^{3}$. Therefore:

- In a mixture of defects of substantially diverse sizes, the smallest defects can be ignored, unless they vastly outnumber the largest ones. This simplifies processing of various data: the information on smallest defects is unnecessary, as far as the effective elastic properties are concerned.

- Since the largest cracks are much larger than the largest pores, they play a dominant role-in spite of a smaller numerical factor for cracks in formulas (2.1).

Thus, it is the microcracks that produce the dominant effect on the effective elastic properties, and only the largest cracks need to be considered. The challenge in their quantitative characterization is that crack geometries are highly "irregular," with partial contacts between crack faces, nonplanarity, and intersected configurations, whereas the usual crack density parameters are defined for flat circular cracks. This issue needs to be addressed; otherwise, crack density becomes a fitting parameter making the effective property-microstructure linkage difficult.

\subsection{Pores}

Pores of the spherical shape are characterized by porosity-relative volume of pores contained in a representative volume $V$ :

$p=\frac{1}{V} \sum V^{(k)}$

where $V^{(k)}$ are the volumes of individual pores. This parameter remains adequate for moderately nonspherical pores (aspect ratios, roughly, between 0.7 and 1.5), provided deviations from the spherical shape are random so that no anisotropy emerges. Indeed, their compliance contribution coincides with that of a set of perfectly spherical pores of the same volume (Ref 17).

Concerning substantially nonspherical pores, we first mention that strongly oblate, crack-like pores (aspect ratios smaller than 0.1 ) can be replaced by cracks, in their impact on the linear elastic and conductive properties. Quantitative characterization of pores that are strongly prolate (aspect ratio $\gamma>1.5$ ) or moderately oblate $(0.1>\gamma>0.7)$ requires certain functions of $\gamma$ (see, for example, Ref 18). However, such pores seem to be less typical for the plasma-sprayed coatings. Generally, pores play a secondary role in the considered coatings, as compared to cracks, and hence their shapes deserve less attention.

\subsection{Cracks}

We consider cracks in greater detail since they constitute microstructural elements of dominant importance. We consider cracks of idealized shapes, irregularly shaped ones, and, importantly, cracks with partial contacts between their faces.

Circular cracks are characterized by the crack density parameter introduced by Bristow (Ref 19):

$\rho=\frac{1}{V} \sum a_{k}^{3}$

where $a_{k}$ is the radius of the $k$ th microcrack. This parameter reflects the fact that compliance contributions of cracks are proportional to $a_{k}^{3}$. Its applicability to cracks of irregular geometries-and its "effective" value in cases when it is applicable-needs to be clarified. This problem has not been fully solved; we review the progress that has been made in this direction.

Parameter $\rho$ can be extended to multiple elliptical cracks with random deviations from circles: with very good accuracy, they can be replaced by an equivalent distribution of the circular cracks, which produces the same impact on the effective elastic properties. Moreover,

. 

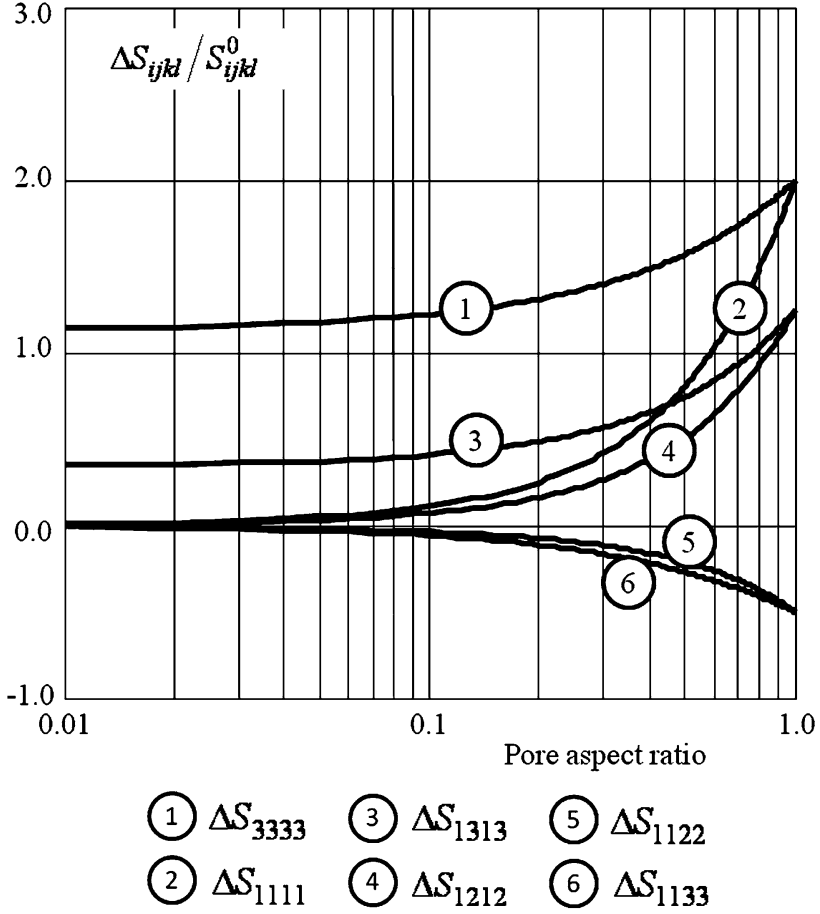

Fig. 3 Compliance contribution components of an oblate pore as functions of pore aspect ratio

the density of the equivalent distribution can be explicitly given in terms of the parameters of the ellipses: the ellipses can be replaced by circles with the same ratios $S^{2} / P$, where $S$ and $P$ are the area and the perimeter, respectively (Ref 20). The approximate equivalence to circular cracks was shown to hold for flat cracks that are irregularly shaped or even intersected in computational study (Ref 21).

Importantly, results for cracks apply to strongly oblate pores as well: their effect on the linear elastic properties (the conductivity-provided heat transfer across cracks is neglected) is approximately the same as the one of cracks, up to aspect ratios of about 0.10 (Fig. 3). Hence, they should be characterized by the crack density-rather than porosity-parameters. Thus,

- Exact information on pore aspect ratios is unnecessary, as long as they are smaller than about 0.10 .

- Porosity is not a relevant parameter for strongly oblate pores. This fact has not been always appreciated in literature (in Ref 9, for example, strongly oblate pores were characterized by their volume fraction). Inadequacy of porosity as a microstructural parameter has been convincingly shown in Ref 4 .

Being a scalar, parameter $\rho$ is appropriate for randomly oriented cracks (overall isotropy) and for perfectly parallel cracks. For more complex orientation distributions, one can use a set of parameters describing the particular distribution (for example, several families of parallel cracks at angles to one another can be described by partial crack densities $\rho_{1}, \rho_{2}, \ldots$ of the families and the angles).
However, for a unified coverage of various orientation distributions, a tensor crack density parameter is needed. Besides providing a unified coverage, it identifies the elastic anisotropy for various orientation distributions.

Thus, a symmetric second-rank crack density tensor is introduced (Ref 22):

$$
\begin{aligned}
\boldsymbol{\alpha} & =\frac{1}{V} \sum\left(a^{3} \boldsymbol{n n}\right)^{(k)}, \text { or, in components, } \\
\boldsymbol{\alpha}_{i j} & =\frac{1}{V} \sum\left(a^{3} n_{i} n_{j}\right)^{(k)}
\end{aligned}
$$

where $\boldsymbol{n}^{(k)}$ is a unit normal to $k$ th microcrack. Its trace $\alpha_{i i}$ is equal to the overall density $\rho$ so that $\alpha$ generalizes the scalar parameter $\rho$. In the case of random orientations, $\alpha_{i j}=(\rho / 3) \delta_{i j}$; for parallel cracks normal to the $x_{3}$-axis, $\alpha=\rho \boldsymbol{e}_{3} \boldsymbol{e}_{3}$; in these simplest cases, tensor $\alpha$ can be replaced by scalar $\rho$. For several families of parallel cracks, $\boldsymbol{\alpha}=\rho_{1} \boldsymbol{n}_{1} \boldsymbol{n}_{1}+\rho_{2} \boldsymbol{n}_{2} \boldsymbol{n}_{2}+\cdots$ where $\boldsymbol{n}_{1}, \boldsymbol{n}_{2} \ldots$ are unit normals to the families.

We emphasize that tensor $\alpha$ is not introduced a priori, but emerges as a result of analysis of individual crack contributions to the overall compliance. This analysis also identifies yet another crack density parameter on which the effective elastic properties depend-the fourth-rank tensor

$\boldsymbol{\beta}=(1 / V) \sum\left(a^{3} \text { nnnn }\right)^{(k)}$

As discussed in Sect 3, the dependence of the elastic properties on $\boldsymbol{\beta}$ is substantially weaker than their dependence on $\boldsymbol{\alpha}$. We mention, nevertheless, its form in several simple cases. In the case of random orientations, $\beta_{i j k l}=(\rho / 15)\left(\delta_{i j} \delta_{k l}+\delta_{i k} \delta_{j l}+\delta_{i l} \delta_{j k}\right)$; for parallel cracks normal to the $x_{3}$-axis, $\boldsymbol{\beta}=\rho \boldsymbol{e}_{3} \boldsymbol{e}_{3} \boldsymbol{e}_{3} \boldsymbol{e}_{3}$; for several families of parallel cracks, $\boldsymbol{\beta}=\rho_{1} \boldsymbol{n}_{1} \boldsymbol{n}_{1} \boldsymbol{n}_{1} \boldsymbol{n}_{1}+\rho_{2} \boldsymbol{n}_{2} \boldsymbol{n}_{2} \boldsymbol{n}_{2} \boldsymbol{n}_{2}+\cdots$.

The characterization by tensor parameters $\alpha$ and $\beta$ can be extended to flat cracks of "irregular" shapes provided the shape irregularities are random (i.e., the distribution of such cracks is equivalent to certain distribution of the circular cracks). However, finding the values of $\boldsymbol{\alpha}$ and $\boldsymbol{\beta}$ remains a challenge (unless cracks have the elliptical shapes).

Cracks in the coatings-particularly the largest ones producing the dominant effect-have a mild tendency to be either parallel to the coating plane $x_{1} x_{2}$ (horizontal cracks) or normal to it (vertical cracks, with normals oriented randomly in the $x_{1} x_{2}$ plane). This results in the transversely isotropic (TI) symmetry, and the principal components of the crack density tensor are $\alpha_{11}=\alpha_{22}$ and $\alpha_{33}$. Both crack families have orientation scatter about the two preferential orientations. This scatter-if it is random-does not violate the TI symmetry, but changes the values of $\alpha_{11}$ and $\alpha_{33}$. We describe the scatter by the following function containing scatter parameter $\lambda$ (which may have different values, $\lambda_{\mathrm{h}}$ and $\lambda_{\mathrm{v}}$, for the horizontal and vertical cracks):

$P_{\lambda}(\varphi)=\frac{1}{2 \pi}\left[\left(\lambda^{2}+1\right) e^{-\lambda \varphi}+\lambda e^{-\lambda \pi / 2}\right]$

The extreme cases of fully random and ideally parallel cracks correspond to $\lambda=0$ and $\lambda=\infty$. Figure 4 shows 


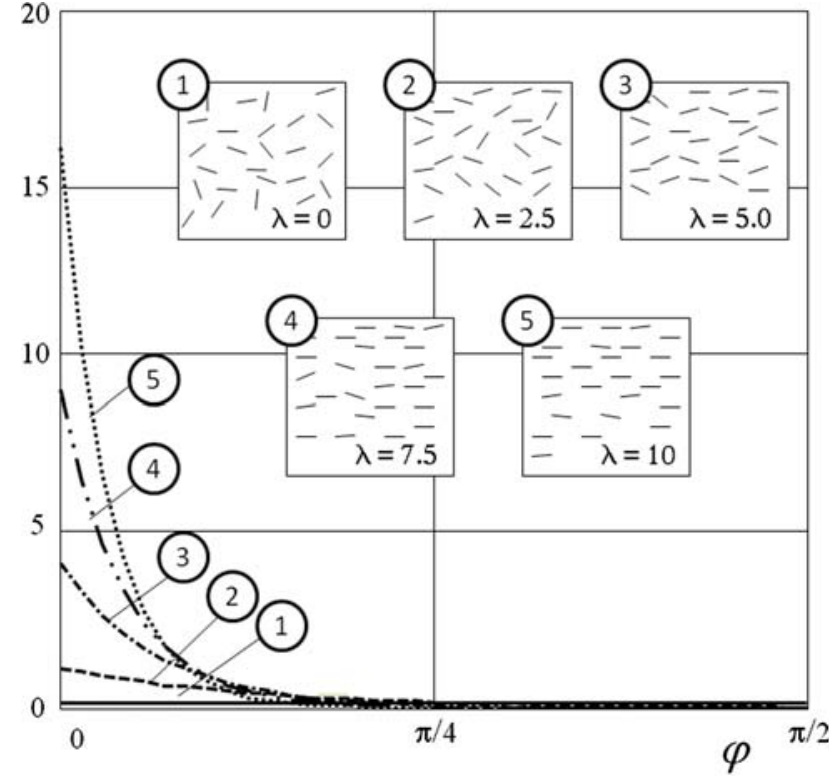

Fig. 4 Dependence of the orientation distribution function $P_{\lambda}$ on angle $\varphi$ at several values of $\lambda$ and the corresponding orientation patterns

orientation patterns corresponding to several values of $\lambda$. All the relevant distribution parameters-partial crack densities $\rho_{\mathrm{h}}$ and $\rho_{\mathrm{v}}$ of the horizontal and vertical cracks and their orientation scatter-are reflected in the values of $\alpha_{11}$ and $\alpha_{33}$ :

$\alpha_{11}=\alpha_{22}=0.5\left[f_{1}\left(\lambda_{\mathrm{v}}\right)+f_{2}\left(\lambda_{\mathrm{v}}\right)\right] \rho_{\mathrm{v}}+f_{1}\left(\lambda_{\mathrm{h}}\right) \rho_{\mathrm{h}}$,

$\alpha_{33}=f_{1}\left(\lambda_{\mathrm{v}}\right) \rho_{\mathrm{v}}+f_{2}\left(\lambda_{\mathrm{h}}\right) \rho_{\mathrm{h}}$

where $f_{1}$ and $f_{2}$ are the functions of the scatter parameter $\lambda$ given by:

$f_{1}=\frac{18-\lambda\left(\lambda^{2}+3\right) e^{-\lambda \pi / 2}}{6\left(\lambda^{2}+9\right)} ; \quad f_{2}=\frac{\left(\lambda^{2}+3\right)\left(3+\lambda e^{-\lambda \pi / 2}\right)}{3\left(\lambda^{2}+9\right)}$

(Eq 2.8)

\subsection{Effect of Contacts Between Crack Faces}

As mentioned above, the contacts-even if they are small-have very strong effect on the compliance and resistance contributions of a crack. The contact "islands" have origin in incomplete cohesion between splats (Fig. 5).

We note that a microcrack-or a plane between layers-may contain multiple contacts. Their analysis is a challenging problem, due to the interactions between contacts that are quite strong and extend over long distances (the effect of a contact decreases as $r^{-1}$ with distance $r$ from the contact center, i.e., quite slowly); they may not be neglected even at spacing between contacts an order of magnitude larger than contact sizes. This makes the interaction effect highly sensitive to mutual positions of contacts. Similar statements hold in the context of conductivity, as demonstrated in Ref 5.

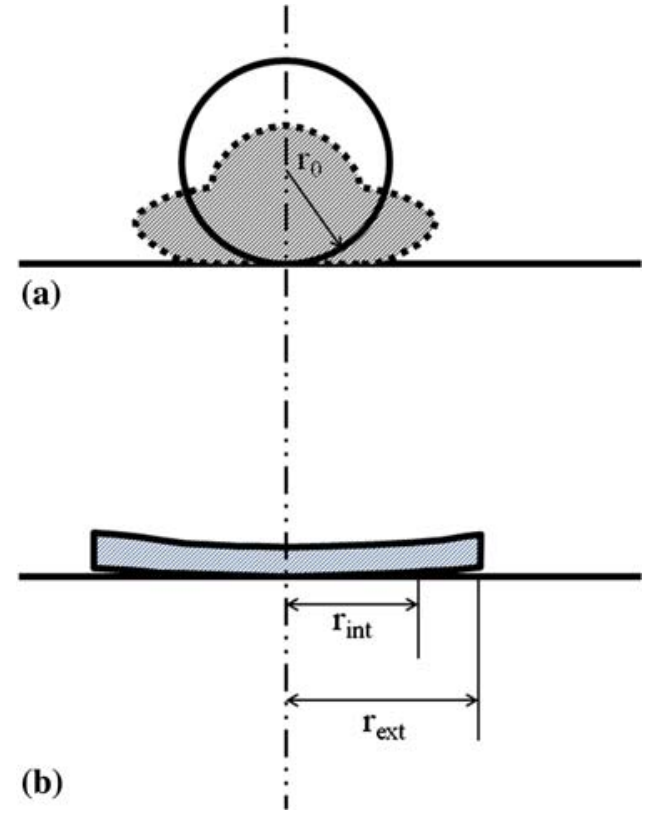

Fig. 5 Splat formation. Adhesion zone at center is surrounded by a debonded zone, resulting in 3-D annular crack geometry (after Ref 6). Its 2-D cross section will show two line cracks

The effect of partial contacts has been analyzed in Ref 15 and 23 using the example of axisymmetric annular crack containing an "island" of cohesion in the middle. The results can be represented in the form of an equivalent circular crack of radius $R_{\text {eff }}$ without an "island" that has the same compliance. An almost vertical drop on the left in Fig. 6 to about $0.70-0.75$ shows that even a very small "island" reduces the crack compliance by a factor of about 2.5 (since crack compliance is proportional to $R_{\mathrm{eff}}^{3}$ ). Thus, cracks with partial contacts can be modeled by cracks of reduced size, and described by appropriately reduced crack density. We add that the exact morphology of the "island"-whether it represents a "welded" area or a Hertzian contact-is irrelevant in the context of the linear elastic response.

The challenge for experimental techniques is to extract information on sizes of "islands"- -this determines the effective radius $R_{\text {eff }}$ and frequency of their occurrence. Note that they may not be easy to detect in 2-D images. For example, two collinear lines may represent two different 3-D geometries: (A) annular crack, with an "island," or (B) two separate cracks (which are not necessarily normal to the plane, and the visible lines do not necessarily represent their largest crosssections). Geometries (A) and (B) may produce very different compliance contributions.

\subsection{On the Quantitative Characterization of "Irregularities" of Crack Shapes}

Microcracks in coatings have "irregular" shapes, and this issue needs to be analyzed, if one does not wish to treat crack density as a fitting parameter thus weakening 


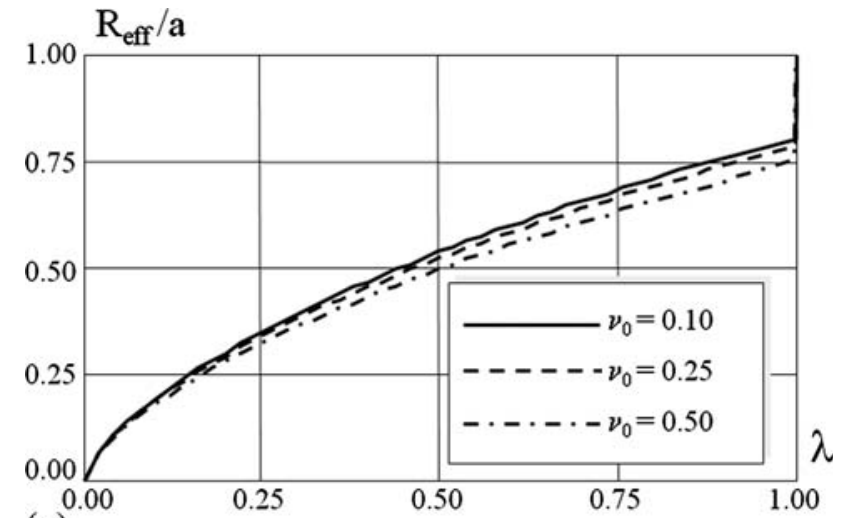

(a)

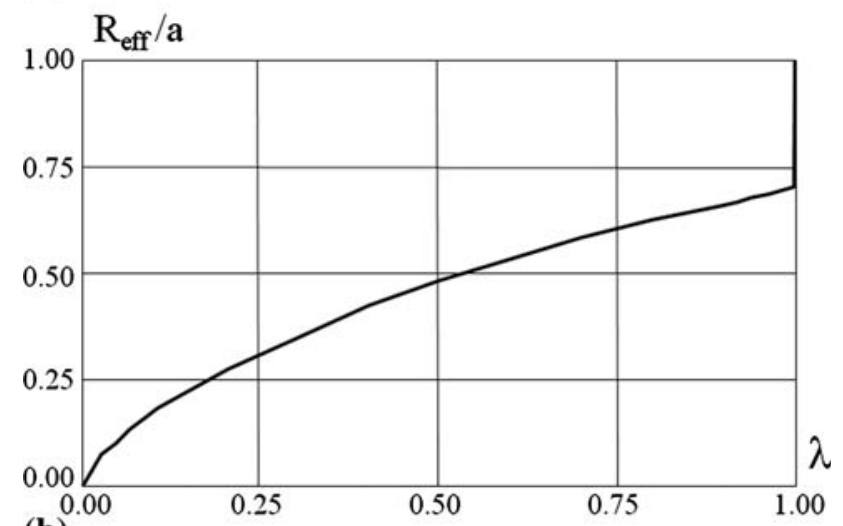

(b)

Fig. 6 (a) Reduction of the crack compliance contribution due to an island. $R_{\text {eff }}$ is a radius of an "equivalent" circular crack (producing the same effect). An almost vertical drop on the left indicates a substantial effect of even a very small island. (b) Analogous reduction of the crack's effect on conductivity in the direction normal to the crack due to an island

the property-microstructure linkage. This problemwhich has to be treated as a 3-D one-has not been fully solved; we briefly review the progress that has been made in this direction.

- Noncircular flat cracks. As mentioned above, multiple cracks of this kind can be replaced by an equivalent distribution of circular cracks, if the deviations from circles are random. If the cracks have approximately elliptical shapes, the equivalence can be established in quantitative terms. For nonelliptical flat cracks, a number of estimates and bounds on crack compliances have been obtained (Ref 23), although they do not cover all possible shapes.

- "Jagged" (corrugated) boundaries of cracks and pores. If jaggedness is slight (its amplitude is much smaller than the defect size), it can be ignored, as far as the effective properties are concerned, and the boundaries can be smoothed out. This follows from bounding the compliance contribution of a pore by the ones of the circumscribed and inscribed "comparison shapes" (Ref 24); in the case of small jaggedness, the bounds are tight. The sensitivity to "jaggedness" is particularly low for narrow, crack-like pores: increasing their aspect ratio to "absorb" the jaggedness and to provide an upper bound would leave the pore compliance almost unchanged, as long as its aspect ratio remains below about 0.1 .

- Nonplanar cracks ("wavy" and "cap" crack patterns). This factor was analyzed in Ref 25 and 26, in the context of elasticity and conductivity, respectively. If nonplanarity is moderate it can be ignored: the crack can be replaced, with satisfactory accuracy, by the planar one of the same area. For strongly nonplanar cracks, a general methodology of estimating their compliance and resistance contributions has been suggested, by replacing the crack by a distribution of small penny-shaped cracks that follow the same orientation distribution as the considered nonflat crack. However, this methodology may lose accuracy for the shapes involving multiple waves.

- Intersected cracks. The impact of intersections on the overall compliance is minimal. As shown by 3-D computational studies (Ref 23), the intersected cracks can be treated as isolated noninteracting ones.

\section{The Effective Elastic Properties of Plasma-Spray Coatings}

We now express the effective elastic properties in terms of proper microstructural parameters identified in the previous section. We represent, as usual, the effective elastic compliances as a sum $S_{i j k l}=S_{i j k l}^{0}+\Delta S_{i j k l}$ where $S_{i j k l}^{0}$ are compliances of the bulk material (assumed to be isotropic) and the change $\Delta S_{i j k l}$ is due to defects.

We first consider cracks-microstructural elements of the dominant importance. In the noninteraction approximation, the compliance change due to them is given in terms of $\boldsymbol{\alpha}$ - and $\boldsymbol{\beta}$-tensors as follows (Ref 20, 22):

$$
\begin{aligned}
\Delta S_{i j k l}=\frac{32\left(1-v_{0}^{2}\right)}{3\left(2-v_{0}\right) E_{0}} & {\left[\frac{1}{4}\left(\delta_{i k} \alpha_{j l}+\delta_{i l} \alpha_{j k}+\delta_{j k} \alpha_{i l}+\delta_{j l} \alpha_{i k}\right)\right.} \\
& \left.-\frac{v_{0}}{2} \beta_{i j k l}\right] .
\end{aligned}
$$

We emphasize that this formula can be used for strongly oblate pores as well.

Of the $\boldsymbol{\alpha}$ - and $\boldsymbol{\beta}$-terms in (3.1), the $\boldsymbol{\alpha}$-term plays the dominant role. Indeed, the $\boldsymbol{\beta}$-term is rooted in the difference between the normal, $B_{\mathrm{N}}$, and shear, $B_{\mathrm{T}}$, compliances of cracks (which relate the average, normal and shear displacement discontinuities, over a crack under applied normal and shear tractions, respectively). Had they been the same (as they are for a 2-D rectilinear crack), the $\boldsymbol{\beta}$-term would have vanished. For the circular crack, $B_{\mathrm{N}}$ and $B_{\mathrm{T}}$ differ by a factor of $1-v_{0} / 2$, i.e., they are relatively close; as a result, the $\boldsymbol{\beta}$-term enters with a relatively small multiplier $v_{0} / 2$. We note that predictions of the effective constants given by formula (3.1) with and 
without the $\boldsymbol{\beta}$-term omitted are quite close and are within typical error margins involved in processing image data and measuring the effective constants. Hence the $\boldsymbol{\beta}$-term can, typically, be neglected and cracks can be characterized solely by the crack density tensor $\boldsymbol{\alpha}$.

The characterization of cracks solely by the crack density tensor $\boldsymbol{\alpha}$ leads to a drastically reduced number of independent elastic constants-from nine, in the general case of orthotropy, to only four, if the orthotropy is due to cracks (regardless of their orientation distribution): all the constants can be expressed in terms of three Young's moduli, $E_{1}, E_{2}, E_{3}$, and one constant of the bulk material $v_{0} / E_{0}$. In the case of transverse isotropy relevant to the coatings ( $x_{3}$ is the symmetry axis), $E_{1}=E_{2}$ and the number of independent effective constants is reduced to only three. In this case, the constants are given in terms of crack density components $\alpha_{11}=\alpha_{22}$ and $\alpha_{33}$ as follows:

$$
\begin{aligned}
& E_{1}=E_{2}=E_{0}\left[1+\frac{32\left(1-v_{0}^{2}\right)}{3\left(2-v_{0}\right)} \alpha_{11}\right]^{-1}, \\
& E_{3}=E_{0}\left[1+\frac{32\left(1-v_{0}^{2}\right)}{3\left(2-v_{0}\right)} \alpha_{33}\right]^{-1} \\
& G_{12}=G_{0}\left[1+\frac{32\left(1-v_{0}\right)}{3\left(2-v_{0}\right)} \alpha_{11}\right]^{-1}, \\
& G_{13}=G_{23}=G_{0}\left[1+\frac{16\left(1-v_{0}\right)}{3\left(2-v_{0}\right)}\left(\alpha_{11}+\alpha_{33}\right)\right]^{-1} \\
& \frac{v_{12}}{E_{1}}=\frac{v_{31}}{E_{3}}=\frac{v_{0}}{E_{0}}
\end{aligned}
$$

Reduction of the number of independent constants to only three is expressed in the following relations showing that the shear moduli are not independent constants:

$\frac{1}{G_{12}}=\frac{1}{E_{1}}+\frac{1}{E_{2}}+\frac{2 v_{0}}{E_{0}}, \quad \frac{1}{G_{13}}=\frac{1}{E_{1}}+\frac{1}{E_{3}}+\frac{2 v_{0}}{E_{0}}$

For the specific transversely isotropic orientation distribution given by (2.6), components $\alpha_{11}$ and $\alpha_{33}$ are given in terms of crack densities of the horizontal and vertical families, $\rho_{\mathrm{h}}$ and $\rho_{\mathrm{v}}$ and their scatter parameters, $\lambda_{\mathrm{h}}$ and $\lambda_{\mathrm{v}}$ by formulas (2.7).

We now modify the expression (3.1) to account for the porosity effect according to the Mori-Tanaka's scheme (MTS) where defects are placed into the average stress, over the solid phase. The average stress increases by a factor of $(1-p)^{-1}$, which enters, therefore, as a multiplier at the compliances given by (3.1). There are two sources of porosity - due to pores (denoted by $p_{\mathrm{p}}$ ) and due to slightly open cracks (denoted by $p_{\mathrm{c}}$ ); the total porosity $p=p_{\mathrm{p}}+p_{\mathrm{c}}$. Hence the MTS modification involves two factors:

- The effect of pores that is be added to the effect of cracks in the framework of NIA, by assuming that pores are placed in the externally applied stress unperturbed by neighbors;

- The amplification effect due to the elevated average stress in the solid matrix; it is described by the amplifying factor $(1-p)^{-1}$ applied to both pores and cracks.
Remark Although slight openings of cracks produce a very minor effect on their compliance contributions (Fig. 3), they affect the overall compliance by contributing to the amplifying factor $(1-p)^{-1}$.

These considerations yield the following modification of (3.1):

$$
\begin{aligned}
\Delta S_{i j k l}= & \frac{32\left(1-v_{0}^{2}\right)}{3\left(2-v_{0}\right) E_{0}} \frac{1}{1-p} \\
& \times\left[\frac{1}{4}\left(\delta_{i k} \alpha_{j l}+\delta_{i l} \alpha_{j k}+\delta_{j k} \alpha_{i l}+\delta_{j l} \alpha_{i k}\right)-\frac{v_{0}}{2} \beta_{i j k l}\right] \\
& +\frac{3\left(1-v_{0}\right)}{2\left(7-5 v_{0}\right) E_{0}} \frac{p_{\mathrm{p}}}{1-p} \\
& \times\left[5\left(1+v_{0}\right)\left(\delta_{i k} \delta_{j l}+\delta_{i l} \delta_{j k}\right)-\left(1+5 v_{0}\right) \delta_{i j} \delta_{k l}\right]
\end{aligned}
$$

where the second term reflects the effect of pores, and the amplifying factor $(1-p)^{-1}$ is applied to both pores and cracks. Applying this formula to the orientation distribution (2.6) we obtain the following modification of formulas (3.2):

$$
\begin{aligned}
& E_{1}=E_{2}=E_{0}\left[1+\frac{32\left(1-v_{0}^{2}\right)}{3\left(2-v_{0}\right)} \frac{\alpha_{11}}{1-p}\right. \\
& \left.+\frac{3\left(1-v_{0}\right)\left(9+5 v_{0}\right)}{2\left(7-5 v_{0}\right)} \frac{p_{\mathrm{p}}}{1-p}\right]^{-1} \\
& E_{3}=E_{0}\left[1+\frac{32\left(1-v_{0}^{2}\right)}{3\left(2-v_{0}\right)} \frac{\alpha_{33}}{1-p}+\frac{3\left(1-v_{0}\right)\left(9+5 v_{0}\right)}{2\left(7-5 v_{0}\right)} \frac{p_{\mathrm{p}}}{1-p}\right]^{-1} \\
& G_{12}=G_{0}\left[1+\frac{32\left(1-v_{0}\right)}{3\left(2-v_{0}\right)} \frac{\alpha_{11}}{1-p}+\frac{15\left(1-v_{0}\right)}{\left(7-5 v_{0}\right)} \frac{p_{\mathrm{p}}}{1-p}\right]^{-1} \\
& G_{13}=G_{23}=G_{0}\left[1+\frac{16\left(1-v_{0}\right)}{3\left(2-v_{0}\right)} \frac{\alpha_{11}+\alpha_{33}}{1-p}\right. \\
& \left.+\frac{15\left(1-v_{0}\right)}{\left(7-5 v_{0}\right)} \frac{p_{\mathrm{p}}}{1-p}\right]^{-1} \\
& \frac{v_{12}}{E_{1}}=\frac{v_{31}}{E_{3}}=\frac{v_{0}}{E_{0}}\left[1+\frac{3\left(1-v_{0}\right)\left(1+5 v_{0}\right)}{2 v_{0}\left(7-5 v_{0}\right)} \frac{p_{\mathrm{p}}}{1-p}\right]
\end{aligned}
$$

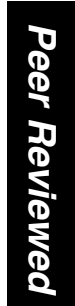

\section{The Conductive Properties and the Cross-Property Connection}

For the effective conductive properties, quantitative characterization of the microstructure is similar-although not identical-to the one for the elastic properties. The key quantity is the resistivity contribution of a defect; the extra resistivity of a representative volume $V$ due to multiple defects is a sum of the individual contributions. In analogy to formulas (2.1) for the elastic compliances, we compare the resistivity contribution $\Delta r$ of the circular crack of radius $a$ placed in a uniform field of heat flux in the direction $x_{1}$ normal to the crack and that of the 
spherical pore of radius $a$, placed in the same field. They are as follows:

$\Delta r_{11}^{\text {crack }}=\frac{8}{3} \frac{1 a^{3}}{k_{0} V} ; \quad \Delta r_{11}^{\text {pore }}=2 \pi \frac{1}{k_{0}} \frac{a^{3}}{V}$

For both of them, the resistivity contribution scales as $a^{3}$. Hence, similar to the elastic compliance contributions,

- The smallest defects can be ignored, unless they vastly outnumber the largest ones;

- Since the largest cracks are much larger than the largest pores, their contribution to the overall resistivity dominates the one of pores (in spite of a larger numerical factor for pores in formula (4.1)).

Similar to the elastic properties, pores can be characterized, with sufficient accuracy, by the porosity parameter $p$ and cracks by the crack density parameters $\alpha_{11}$ and $\alpha_{33}$. Importantly, the effect of partial contacts between crack faces on the crack resistivity contribution coincides, to within a multiplier, with their effect on the crack compliance contribution (Ref 15), see Fig. 6 (this follows, as exact result, from the cross-property connections for imperfect interfaces, Ref 27). The shape "irregularity factors" that produce only a minor effect on the elastic properties - such as "jaggedness" of the pore boundaries or mild nonplanarity of cracks-remain unimportant for conductivity as well.

However, there are differences between the two properties in the case of anisotropy. For the elastic properties, the orthotropic symmetry has approximate character: it hinges on neglecting the contribution of the fourth-rank microstructural tensor $\boldsymbol{\beta}=(1 / V) \sum\left(a^{3} \text { nnnn }\right)^{(k)}$ in (3.1) or (3.4). In contrast, the conductive properties are described by a symmetric second-rank conductivity tensor, and hence they are always rigorously orthotropic; fourth-rank microstructural tensors are irrelevant for them.

Remark Obviously, the exact orthotropic symmetry of the conductive properties does not rule out approximate character of higher symmetries-the transverse isotropy or isotropy - that may hold in special cases of orientation distribution of defects (for example, if the distribution is approximately axisymmetric or approximately random).

Using the same micromechanics-based approach as in the case of the elastic compliances, we obtain effective conductivities in terms of $\alpha_{11}, \alpha_{33}$, and porosity, in the form that is analogous to (3.5):

$k_{1}=k_{2}=k_{0}\left[1+\frac{8 \alpha_{11}}{3(1-p)}+\frac{3}{2} \frac{p_{\mathrm{p}}}{1-p}\right]^{-1} ;$

$k_{3}=k_{0}\left[1+\frac{8 \alpha_{33}}{3(1-p)}+\frac{3}{2} \frac{p_{\mathrm{p}}}{1-p}\right]^{-1}$,

where the amplifying factor $(1-p)^{-1}$ represents the Mori-Tanaka correction for the interaction effect analogous to the one for the elastic properties-the increase in the average temperature gradient over the solid phase under the same applied heat flux.
The similarity between the microstructural parameters that control the elastic and the conductive properties leads to explicit cross-property connection between the two properties. The proportionality factor entering these connections generally depends on the average shapes of inhomogeneities (Ref 28). In particular, for cracks of any orientation distribution, the connection has the form:

$\frac{E_{0}-E_{i}}{E_{i}} \approx \underbrace{\frac{4\left(1-v_{0}^{2}\right)}{2-v_{0}}}_{2.14 \text { at } v_{0}=0.3} \frac{k_{0}-k_{i}}{k_{i}}$

It relates changes in Young's modulus and in conductivity in the same direction $x_{i}$. In practice, one is usually interested in the conductivity $k_{3}$ normal to the coating and in the stiffness $E_{1}$ parallel to it.

This connection is universal-it holds in any direction $x_{i}$ and applies to all orientation distributions of cracks. At the same time, it is approximate since it is based on neglecting the contribution of fourth-rank tensor $\boldsymbol{\beta}$. We mention that in two special cases of orientation distribution, ideally parallel and randomly oriented cracks, more accurate results can be given since the mentioned contribution does not have to be neglected in these two cases. Namely, for parallel cracks, in the direction normal to cracks,

$\frac{E_{0}-E_{i}}{E_{i}}=\underbrace{2\left(1-v_{0}^{2}\right)}_{1.82 \text { at } v_{0}=0.3} \frac{k_{0}-k_{i}}{k_{i}}$

and, for randomly oriented cracks,

$\frac{E_{0}-E}{E}=\underbrace{\frac{2\left(1-v_{0}^{2}\right)\left(10-3 v_{0}\right)}{5\left(2-v_{0}\right)}}_{1.95 \text { at } \mathrm{v}_{0}=0.3} \frac{k_{0}-k}{k}$

These results indicate that the actual cross-property factor for complex orientation distributions-such as the ones observed in coatings-is likely to be slightly lower than the one entering (4.3).

The utility of the cross-property connection is seen as follows:

- it identifies possible combinations of the elastic/ conductive properties;

- it may be useful if one of the two properties is easier to measure than the other one;

- It provides a "control tool" for various micromechanics models, by requiring that models for the elastic properties should be compatible with the ones for the conductive properties.

\section{Modeling of YSZ Coatings: Case Studies}

We now examine experimental data available in literature in light of the micromechanics-based modeling 
outlined in Sect 2-4. The following goals were pursued:

- To demonstrate that the theory can directly process the microstructural data;

- To affirm the ability of the model to predict the elastic and the conductive properties in terms of microstructure, as given by Eq 3.5 and 4.2;

- To verify the cross-property connection (4.3);

- To identify uncertainties in extraction of the needed microstructural information from 2-D photomicrographs.

The microstructure-property connections and the crossproperty linkage (formula (4.3)) are normalized to the bulk material constants, $E_{0}, v_{0}$, and $k_{0}$. Therefore, their values should correspond to the specific material used in a considered experiment-as was done in Ref 16 and 29 discussed in the text to follow. This is important in view of substantial variation in $E_{0}$ and $k_{0}$ (depending on the processing parameters, primarily, due to the extent of crystallinity and the crystalline structure, the yttria content, etc.) that has been reported in literature: for YSZ, the reported values of $k_{0}$ vary from 1.8 to $3.5 \mathrm{~W} / \mathrm{mK}$ and for $E_{0}$ from 180 to $210 \mathrm{GPa}$ (see, for example, Ref 30 and information available at the following Web sites: http:// www.crystec.de/daten/ysz.pdf; http://www.stanfordmaterials. com/media.html). However, many of the published data on the effective values of $E$ and $k$ take the bulk constants, $E_{0}$ and $k_{0}$, from one of the literature sources, without discussing their relevance to the particular experiment. Such uncertainty in the bulk constants leads to large variations of the "apparent" cross-property factor. For example, the data of Ref 31 (their Table 1) on the effective values of $E$ and $k$ implies variation of the "apparent" factor from 0.5 to 2 , if $E_{0}$ and $k_{0}$ are varied in the abovementioned interval. In addition, some of the published data do not make it clear whether $E$ and $k$ were measured in the same direction or not.

We now discuss results of Ref 16 where the bulk constants were taken for the specific material used in coatings. The bulk material constants $E_{0}=210 \mathrm{GPa}, v_{0}=0.3$, and $k_{0}=2.2 \mathrm{~W} / \mathrm{mK}$ corresponded to the specific processing conditions under which the coatings had been manufactured at General Electric facilities. As discussed in Sect 2, the coating is characterized by two crack densities $\alpha_{11}$ and $\alpha_{33}$ and porosity $p$, the latter playing a secondary role. Their values had to be extracted from $2-\mathrm{D}$ image data. Whereas estimation of $p$ was relatively reliable, determination of $\alpha_{11}$ and $\alpha_{33}$ involved substantial uncertainties. Fortunately, smaller microcracks could be ignored since contributions of individual cracks to $\alpha_{11}$ and $\alpha_{33}$ (and hence to the overall properties) are proportional to their sizes cubed.

The main microstructural uncertainty-and hence the difficulty in estimating $\alpha_{11}$ and $\alpha_{33}$-was in extraction of information on "islands" of partial contacts between crack faces. For example, two collinear crack lines in a cross section can be interpreted either as traces of two isolated cracks or as a trace of a larger annular crack with "islands" of partial contact in the middle; the latter interpretation would yield substantially higher values of $\alpha_{11}$ and $\alpha_{33}$. We dealt with these uncertainties by first assuming that each line corresponds to one isolated crack and then introduced a correction factor for the "islands"; to this end, we used one of the specimens for calibration and then applied the calibration factor to other specimens. As discussed below, this calibration factor had a stable value, from one specimen to another.

\subsection{Verification of the Microstructure-Property Relationships}

To verify relations (3.5) and (4.2), we processed data from photomicrographs of four specimens. We first determined the above-mentioned calibration factor for "islands" of partial contacts from specimen A. If all the crack lines in the photomicrograph of this specimen A are interpreted as traces of isolated cracks, then the estimated values of crack densities are $\alpha_{11}=0.14$ and $\alpha_{33}=0.10$; Eq 3.5 and 4.2 would then yield $E_{1}=111 \mathrm{GPa}$ and $k_{3}=1.69 \mathrm{~W} / \mathrm{mK}$. That is a very substantial overestimation of the experimental data given in Table 1. The disagreement was interpreted as an indication that a significant proportion of the traces represented larger annular cracks so that $\alpha_{11}$ and $\alpha_{33}$ had to be multiplied by a certain factor. The latter depends on the average ratio of the internal to external radii of the annular cracks-information that could not be directly estimated from the photomicrographs. We chose the calibration factor of 4.6 to match the conductivity and elasticity data for the specimen $\mathrm{A}$; this factor incorporates, in an integral way, both the effect of partial contacts between the crack faces and the transition from two-dimensional images to three-dimensional crack densities. Then, the "effective" crack densities for the specimen A are: $\alpha_{11}=0.46$ and $\alpha_{33}=0.32$ (see Table 2 where the values of $\alpha_{11}$ and $\alpha_{33}$ are also given). These values yield $E_{1}=53.6 \mathrm{GPa}$ and $k_{3}=1.13 \mathrm{~W} / \mathrm{mK}$-in

Table 1 Effective properties of four specimens presented in Fig. 7 and 8

\begin{tabular}{lccccc}
\hline & \multicolumn{3}{c}{$\boldsymbol{E}_{\mathbf{1}}, \mathbf{G P a}$} & & \multicolumn{1}{c}{$\boldsymbol{k}_{\mathbf{3}}, \mathbf{W} / \mathbf{m K}$} \\
\cline { 2 - 5 } Specimen & Measured & Formula (3.5) & Cross-property formula (4.3) & & Measured \\
\hline (A) & $51.0 \pm 5.1$ & 53.6 & 56.98 & $1.16 \pm 0.16$ \\
(B) & $44.1 \pm 4.4$ & 41.5 & 47.02 & $1.02 \pm 0.14$ \\
(C) & $34.2 \pm 3.4$ & 35.3 & 33.64 & $0.80 \pm 0.11$ \\
(D) & $18.6 \pm 1.9$ & 22.25 & 20.11 & $0.53 \pm 0.07$
\end{tabular}

Direct influence of porosity is ignored in calculations 
agreement with the data of Table 1. Importantly, both the elastic modulus and the conductivity were matched-an indication of correctness of the underlying microstructural model.

In further support of the value of 4.6 of the calibration factor, we note that it corresponds to the ratio 0.5 of the internal to external radii of annular cracks. This number agrees with the data given in the book (Ref 6), where the mentioned ratio was estimated to be in the range 0.4-0.7.

We applied the calibration factor of 4.6 to the other three specimens when processing the photomicrographical data. Then the predicted values of the elastic modulus and the conductivity for these specimens (Table 1) agreed quite well with the experimental data for all the specimens.

\subsection{Verification of the Elastic-Conductive Cross-Property Connection}

The main difficulty in the verification was that the available data on the elastic modulus and on the conductivity were in different directions-normally and parallel to the substrate $\left(E_{1}\right.$ and $k_{3}$ ), whereas the connection relates the stiffness and the conductivity in the same direction. Therefore, we had to verify the connection indirectly, by estimating the ratio $\alpha_{11} / \alpha_{33}$ that characterizes the extent of anisotropy of microcrack orientations. In terms of this ratio, we could estimate $\alpha_{33}$ by extracting the

Table 2 Microstructural characteristics of four specimens presented in Fig. 7 and 8

\begin{tabular}{lcccc}
\hline Specimen & $\boldsymbol{\rho}, \mathbf{g} / \mathbf{c m}^{\mathbf{3}}$ & $\boldsymbol{p}$ & $\boldsymbol{\alpha}_{\mathbf{1 1}}\left(\mathbf{=} \boldsymbol{\alpha}_{\mathbf{2 2}}\right)$ & $\boldsymbol{\alpha}_{\mathbf{3 3}}$ \\
\hline (A) & $5.42 \pm 0.34$ & 0.103 & 0.46 & 0.32 \\
(B) & $5.32 \pm 0.58$ & 0.121 & 0.64 & 0.46 \\
(C) & $5.29 \pm 0.06$ & 0.126 & 0.78 & 0.55 \\
(D) & $5.25 \pm 0.27$ & 0.132 & 1.33 & 0.95
\end{tabular}

The data on density is used for estimation of porosity (the density of the virgin material $\rho_{0}=6.05 \mathrm{~g} / \mathrm{cm}^{3}$ )
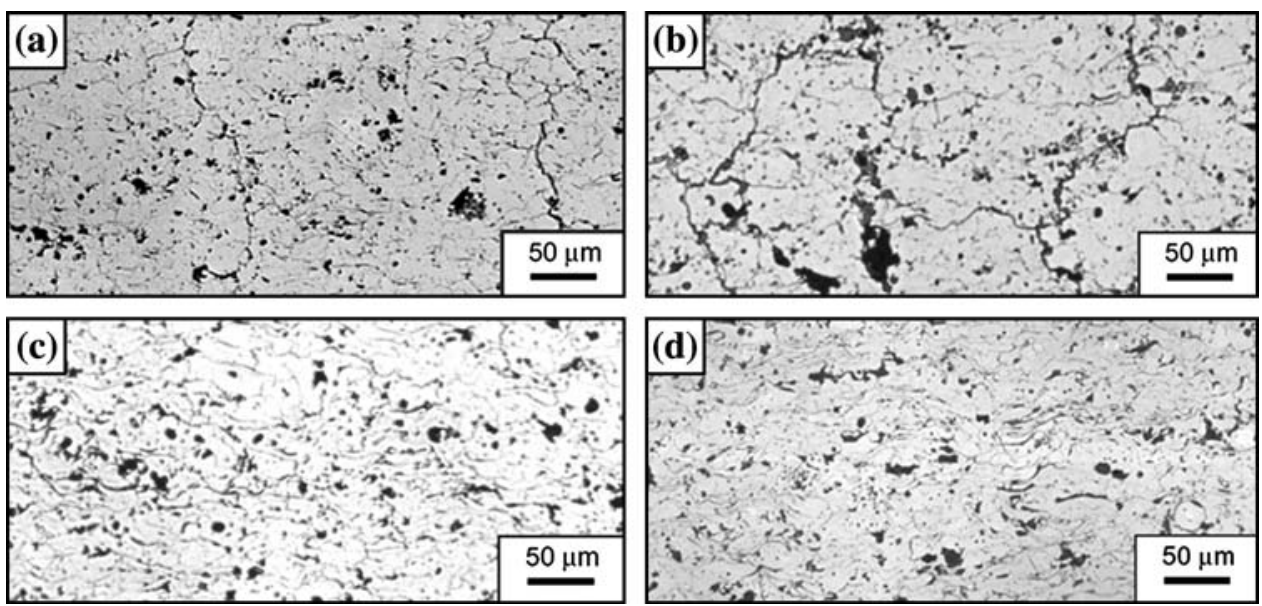

Fig. 7 Microstructures of four YSZ plasma-sprayed coatings that have been processed in the framework of the developed model

value of $\alpha_{11}$ from the data on $E_{1}$ and hence predict $k_{3}$. Then the cross-property connection (4.3) takes the form:

$\frac{E_{0}-E_{1}}{E_{1}}=\frac{4\left(1-v_{0}^{2}\right)}{2-v_{0}} \frac{\alpha_{11}}{\alpha_{33}} \frac{k_{0}-k_{3}}{k_{3}}$

The ratio $\alpha_{11} / \alpha_{33}$ was estimated from photomicrographs (see Fig. 7 and 8 ) by interpreting the crack lines as radii of circular cracks (ignoring the calibration factor for "islands" assuming that the factor applies equally to microcracks of all orientations). This ratio was about 1.4 for all four specimens (a moderate dominance of the vertical microcracks, due, mostly, to large vertical cracks).

Table 1 shows good agreement between the data and the values predicted by the connection. The maximal error was $12 \%$ for specimen $\mathrm{B}$; for the other three specimens, the error did not exceed $8 \%$. Importantly, the agreement takes place in spite of the fact that reductions in the values of $E_{1}$ and $k_{3}$ as compared to the bulk material were very large-by a factor of 4-5.

\section{Discussion and Conclusions}

Various approaches to linking the effective elastic and conductive properties of the plasma-sprayed ceramic coatings to their microstructure have been discussed. In particular, we emphasized equivalence of two approaches to modeling the microstructure: the one based on the statistics of contacts between splats and the microcrackbased one (provided partial contacts between crack faces are reflected in the "effective" values of the microcrack density).

The microcrack-based approach appears to be preferable since it provides a convenient framework for processing 2-D image data where the largest microcrackswhich produce the main effect on the overall properties - can be identified. Also, by utilizing the effective media models for cracked materials, it provides a framework for predicting the full set of anisotropic effective 

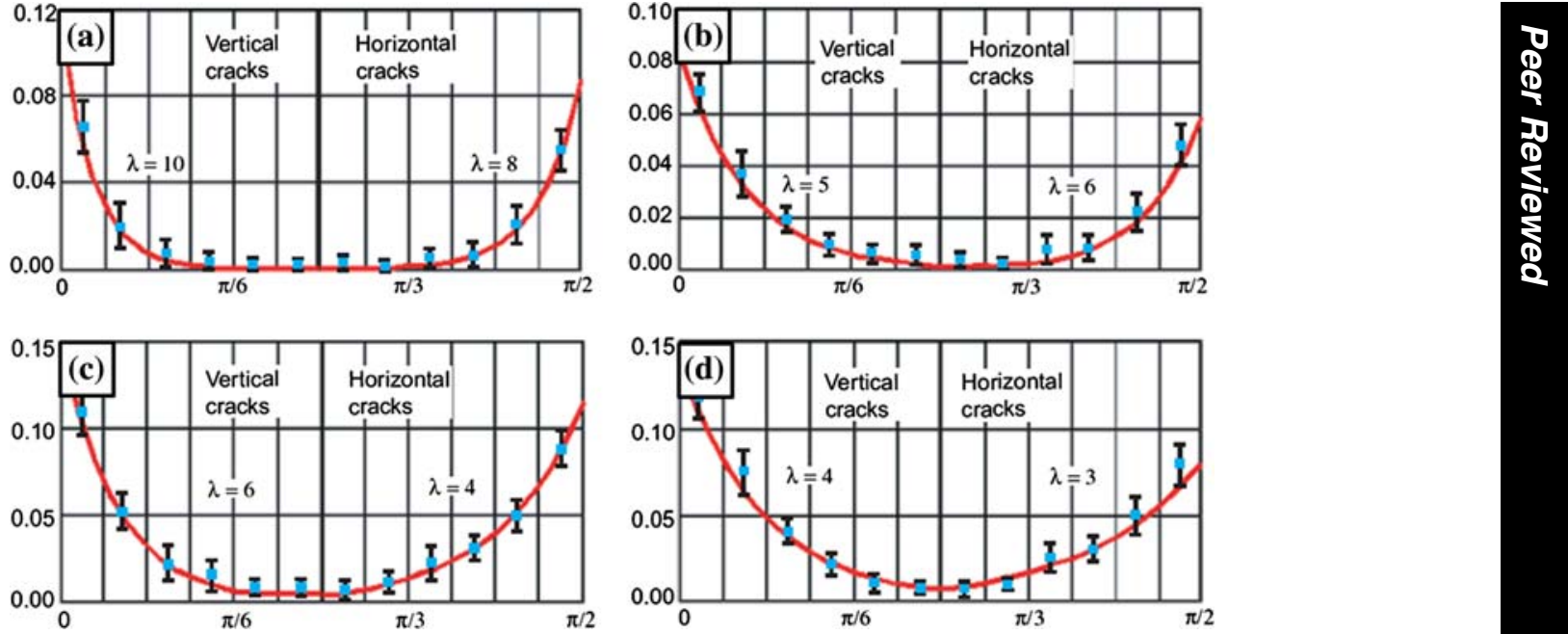

Fig. 8 Two-dimensional crack densities extracted from images of microstructures of the four specimens shown in Fig. 7
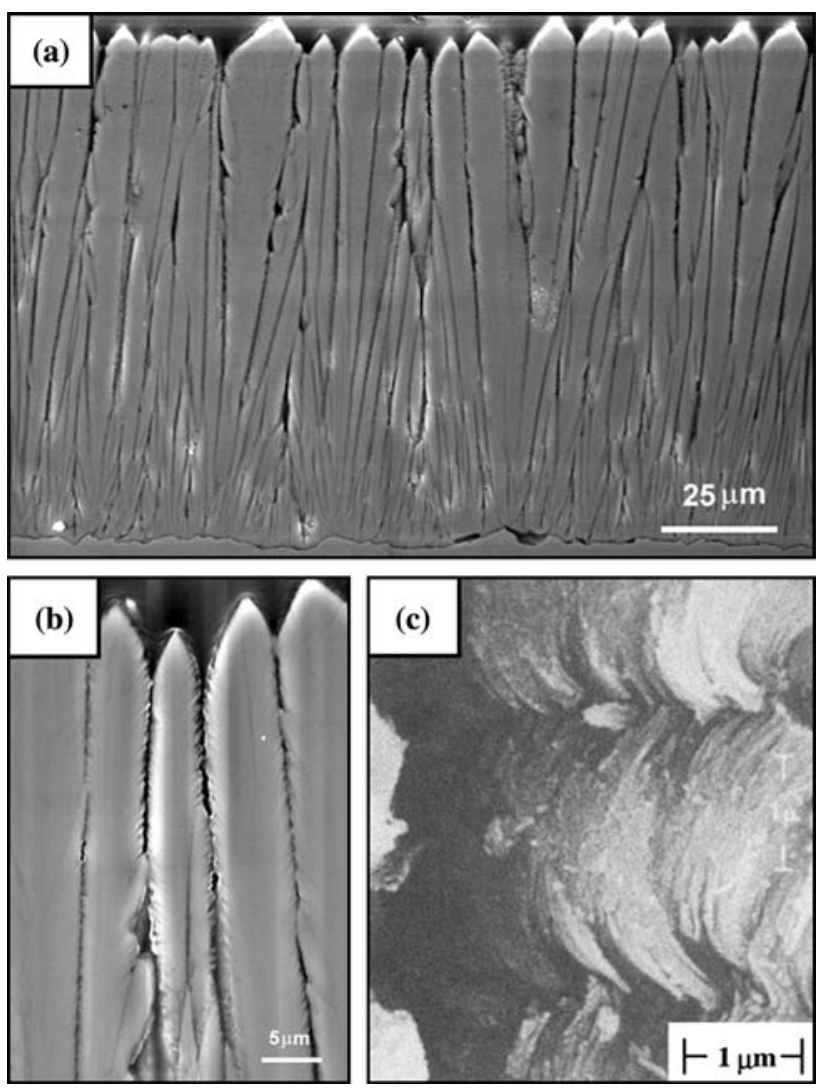

Fig. 9 Typical microstructure of PVD ceramic coatings at different magnifications. Note multiple contacts between the microstructural elements

constants. In the case of transverse isotropy that is relevant for the coatings considered, the controlling microstructural parameters are two principal values of the crack density tensor-microcrack densities $\alpha_{11}$ and $\alpha_{33}$ that reflect, in an integral way, the density of microcracks and their orientation scatter about the "horizontal" and "vertical" directions. Their values also reflect partial contacts between crack faces that substantially reduce the "effective" crack density. Both the elastic and the conductive properties are expressed in their terms. Importantly, these results apply to strongly oblate pores (aspect ratios below 0.1 ) as well. The porosity parameter $p$ usually plays a secondary role (at least at porosities not exceeding $10-15 \%)$; in the framework of the Mori-Tanaka's scheme, it enters as a correction multiplier $(1-p)^{-1}$ that amplifies the effect of microcracks.

One of the advantages of identifying the proper microstructural parameters $\left(\alpha_{11}, \alpha_{33}\right.$, and $\left.p\right)$ is that they imply cross-property connections between the elastic and the conductive properties. They may be useful if one of the properties is easier to measure than the other one. Besides, they provide a "control tool," by requiring that models for the elastic properties should be compatible with the ones for the conductive properties.

We discussed difficulties in processing the microstructural information provided by 2-D image photomicrographical data and related uncertainties. One of them is in the extraction of information on partial contacts between crack faces-the factor of primary importance that determines the reduced "effective" value of microcrack densities.

Remark on the PVD coatings Although the present review focuses on the plasma-sprayed coatings, we comment on modeling of the physical vapor deposition (PVD) ones, where one of the quantities of interest is the elastic stiffness in directions parallel to the coating (in connection with thermal expansion of the substrate). The microstructure of PVD coatings (Fig. 9) differs from the one of the plasma-spray coatings in an essential way-it cannot be modeled as a continuous material containing isolated cracks and pores. Instead, it is formed by multiple contacts along rough surfaces, the latter crossing the entire "representative volume." This implies that the microstructural 
characterization must be different-it should reflect the distribution of contacts (Ref 32). An attempt to model the PVD coating by a continuum with pores (for example, needle-like ones) leads to gross overestimation of the mentioned stiffness (Ref 33).

\section{References}

1. M. Kachanov and I. Sevostianov, On Quantitative Characterization of Microstructures and Effective Properties, Int. J. Solids Struct., 2005, 42, p 309-336

2. F. Kroupa, Nonlinear Behavior in Compression and Tension of Thermally Sprayed Ceramic Coatings, J. Therm. Spray Technol., 2007, 16, p 84-95

3. R. McPherson, Model for the Thermal Conductivity of PlasmaSprayed Ceramic Coatings, Thin Solid Films, 1984, 112, p 89-95

4. C.-J. Li and A. Ohmori, Relationships Between the Microstructure and Properties of Thermally Sprayed Deposits, J. Therm. Spray Technol., 2002, 11, p 365-374

5. J.A. Greenwood, Constriction Resistance and the Real Area of Contact, Brit. J. Appl. Phys., 1966, 17, p 1621-1632

6. V.V. Kudinov, Plasma Sprayed Coatings, Nauka, Moscow, 1977 ((in Russian))

7. L. Pawlowski, The Science and Engineering of Thermal Sprayed Coatings, J. Wiley, Chichester, 1995

8. F. Kroupa, Effect of Nets of Microcracks on Elastic Properties of Materials, Kovove Mater., 1995, 33, p 418-426

9. S.-H. Leigh and C. Berndt, Modelling of Elastic Constants of Plasma-Spray Deposits with Ellipsoid-Shaped Voids, Acta Mater., 1999, 47, p 1575-1586

10. F. Kroupa and M. Kachanov, Effect of Microcracks and Pores on the Elastic Properties of Plasma Sprayed Materials, Proc. 19-th Int. Symp. on Materials Science, J.V. Carstensen, et al., Ed., Riso National Lab, Roskilde, Denmark, 1998, p 325-330

11. I. Sevostianov and M. Kachanov, Modeling of the Anisotropic Elastic Properties of Plasma-Sprayed Coatings in Relation to Their Microstructure, Acta Mater., 2000, 48, p 1361-1370

12. I. Sevostianov and M. Kachanov, Anisotropic Thermal Conductivities of Plasma-Sprayed Thermal Barrier Coatings in Relation to the Microstructure, J. Therm. Spray Technol., 2000, 9(4), p 478482

13. I. Sevostianov and M. Kachanov, Plasma Sprayed Ceramic Coatings: Anisotropic Elastic and Conductive Properties in Relation to the Microstructure. Cross-Property Correlations, Mater. Sci. Eng., 2001, 297, p 235-243

14. I.O. Golosnoy, S.A. Tsipas, and T.W. Clyne, An Analytical Model for Simulation of Heat Flow in Plasma-Sprayed Thermal Barrier Coatings, J. Therm. Spray Technol., 2005, 14, p 205-214

15. I. Sevostianov, Explicit Relations Between Elastic and Conductive Properties of a Material Containing Annular Cracks, Philos. Trans. R. Soc. A, 2003, 361, p 987-999

16. I. Sevostianov, M. Kachanov, J. Ruud, P. Lorraine, and M. Dubois, Quantitative Characterization of Microstructures of
Plasma-Sprayed Coatings and Their Conductive and Elastic Properties, Mater. Sci. Eng. A, 2004, 386, p 164-174

17. M. Kachanov, I. Tsukrov, and B. Shafiro, Effective Moduli of Solids with Cavities of Various Shapes, Appl. Mech. Rev., 1994, 47(1), p S151-S174

18. I. Sevostianov and M. Kachanov, Explicit Cross-Property Correlations for Anisotropic Two-Phase Composite Materials, J. Mech. Phys. Solids, 2002, 50, p 253-282

19. J.R. Bristow, Microcracks, and the Static and Dynamic Elastic Constants of Annealed Heavily Cold-Worked Metals, Brit. J. Appl. Phys., 1960, 11, p 81-85

20. M. Kachanov, Effective Elastic Properties of Cracked Solids: Critical Review of Some Basic Concepts, Appl. Mech. Rev., 1992, 45(8), p 304-335

21. V. Grechka and M. Kachanov, Effective Elasticity of Fractured Rocks: A Snapshot of the Work in Progress, Geophysics, 2006, 71, $\mathrm{p}$ W45-W58

22. M. Kachanov, Continuum Model of Medium with Cracks, J. Eng. Mech. Div.-ASCE, 1980, 106, p 1039-1051

23. I. Sevostianov and M. Kachanov, On Elastic Compliances of Irregularly Shaped Cracks, Int. J. Fract., 2002, 114, p 245-257

24. R. Hill, Elastic Properties of Reinforced Solids: Some Theoretical Principles, J. Mech. Phys. Solids, 1963, 11, p 357-372

25. M.E. Mear, I. Sevostianov, and M. Kachanov, Elastic Compliances of Non-Flat Cracks, Int. J. Solids Struct., 2007, 44(2007), p 6412-6427

26. M. Kachanov, M.E. Mear, J. Rungamornrat, and I. Sevostianov, Resistances of Non-Flat Cracks, and Their Relation to Crack Compliances, Int. J. Eng. Sci., 2009, 47, p 754-766

27. J.R. Barber, Bounds on the Electrical Resistance Between Contacting Elastic Rough Bodies, Proc. R. Soc. Lond. A, 2003, 459, p 53-66

28. I. Sevostianov and M. Kachanov, Connections Between Elastic and Conductive Properties of Heterogeneous Materials, Advances in Applied Mechanics, E. van der Giessen and H. Aref, Ed., Academic Press, 2008, Vol. 42, p 69-252

29. I. Sevostianov, M. Kachanov, J. Ruud, P. Lorraine, and M. Dubois, Micromechanical Analysis of Plasma Sprayed TBC: Anisotropic Elastic and Conductive Properties in terms of Microstructure. Experimental Verification on YSZ Coatings, Thermal Spray 2003: Advancing the Science and Applying the Technology, B.R. Marple and C. Moreau, Ed., ASM International, Materials Park, OH, 2003, p 1557-1563

30. R. Vassen, X. Cao, F. Tietz, D. Basu, and D. Stöver, Zirconates as New Materials for Thermal Barrier Coatings, J. Am. Ceram. Soc., 2004, 83, p 2023-2028

31. Z. Wang, A. Kulkarni, S. Deshpande, T. Nakamura, and H. Herman, Effects of Pores and Interfaces on Effective Properties of Plasma Sprayed Zirconia Coatings, Acta Mater., 2003, 51, p 5319-5334

32. I. Sevostianov, M. Kachanov, and J. Ruud, On the Elastic Properties of PVD Coatings in Relation to Their Microstructure, ASME J. Eng. Mater. Technol., 2002, 124, p 246-249

33. T.J. Lu, C.G. Levi, H.N.G. Wadley, and A.G. Evans, Distributed Porosity as a Control Parameter for Oxide Thermal Barriers Made by Physical Vapor Deposition, J. Am. Ceram. Soc., 2001, 84, p 2937-2946 